Cinémas

Revue d'études cinématographiques

Journal of Film Studies

\title{
Le cinéma québécois aux États-Unis a-t-il plus de chances d'être mieux reçu en anglais?
}

\section{Pierre Véronneau}

Volume 7, numéro 3, printemps 1997

Cinéma québécois et États-Unis

URI : https://id.erudit.org/iderudit/1000950ar

DOI : https://doi.org/10.7202/1000950ar

Aller au sommaire du numéro

Éditeur(s)

Cinémas

ISSN

1181-6945 (imprimé)

1705-6500 (numérique)

Découvrir la revue

Citer cet article

Véronneau, P. (1997). Le cinéma québécois aux États-Unis a-t-il plus de chances d'être mieux reçu en anglais? Cinémas, 7(3), 81-118.

https://doi.org/10.7202/1000950ar
Résumé de l'article

L'accueil de la production québécoise anglophone dans la presse régulière et corporative des États-Unis évolue selon les époques. Selon qu'il s’agisse de cinéma d'auteur ou de genre, ou du travail de cinéastes étrangers, les opinions varient. Cette réception permet de nuancer les prétentions internationales anglophones du cinéma québécois, car l'accueil à l'étranger est toujours fonction des critères valorisés par l'institution d'interprétation et non des paramètres déterminés par le pays d'origine. 


\title{
Le cinéma québécois aux États-Unis a-t-il plus de chances d'être mieux reçu en anglais?
}

\section{Pierre Véronneau}

\begin{abstract}
RÉSUMÉ
Laccueil de la production québécoise anglophone dans la presse régulière et corporative des États-Unis évolue selon les époques. Selon qu'il s'agisse de cinéma d'auteur ou de genre, ou du travail de cinéastes étrangers, les opinions varient. Cette réception permet de nuancer les prétentions internationales anglophones du cinéma québécois, car l'accueil à l'étranger est toujours fonction des critères valorisés par l'institution d'interprétation et non des paramètres déterminés par le pays d'origine.
\end{abstract}

\section{ABSTRACT}

The reception of Anglophone Quebec productions in the mainstream American corporate press has changed through the years. Depending on whether it is an authorial or genre production, or seen as the work of foreign filmmakers, opinions have varied. This reception makes it possible to question the international Anglophone pretensions of Quebec cinema because its reception abroad is always determined by the criteria of the interpreting institutions and not by parameters set by the producing country.

La majorité des textes publiés sur le cinéma québécois, ici et à l'étranger, s'arrête à des œuvres et à des films valorisés pour leurs 
qualités artistiques, intellectuelles et culturelles. Ceci a pour résultat la récurrence régulière de certains noms et de certains titres qui s'imposent et atteignent même le statut de canons. Cependant, lorsqu'on confronte cet ensemble de films avec les répertoires publiés par la Cinémathèque québécoise' ou encore avec l'Index des films canadiens de long métrage, force est de constater qu'ils ne correspondent, surtout après 1970, qu'à une proportion restreinte de ce que les répertoires entendent comme production québécoise ${ }^{2}$. Qui plus est, la plupart des textes critiques auxquels je viens de faire référence traitent surtout de films francophones. Seuls quelques films anglophones, ceux de Paul Almond ou de Robin Spry, par exemple, intéressent un tant soit peu la critique.

Quand on étudie les deux répertoires analytiques publiés par le Centre de recherche cinéma/réception de l'Université de Montréal (CRC/R) sur les films québécois dans la critique américaine, on ne peut manquer de noter les quelque 130 longs métrages anglophones qui s'y retrouvent - un nombre important, expliqué par la communauté de langue diront certains. Parmi ceux-ci, les œuvres significatives du point de vue de la culture nationale (ou disons, puisque ce concept peut être difficile à cerner, du point de vue du consensus intellectuel et critique tel que le reflètent les livres et les articles canadiens sur le cinéma) se retrouvent dans une minorité des textes critiques recensés. On peut déjà deviner que mon étude portera, elle, sur ces films qui jouissent au contraire d'une réputation souvent douteuse et qui ont peu retenu l'attention d'individus appartenant à ce que Bordwell (1989) appelle l'institution d'interprétation et cela, malgré le fait que quelques-uns d'entre eux aient été des succès de fréquentation.

Dans l'ensemble de ce corpus, j'ai tout de même procédé à quelques choix; j'ai exclu les documentaires, les films d'animation et les films de l'ONF entièrement tournés hors Québec sur des sujets non québécois. En considérant l'emplacement du siège social de l'ONF à Montréal comme un critère insuffisant à la retenue des films de cette société d'État dans mon étude, je sais que je me place en porte à faux. Mais il m'apparaissait davantage significatif de ne retenir que les films anglophones 
tournées par l'industrie privée au Québec et d'analyser les stratégies économiques et commerciales qui sous-tendent ces productions. L'analyse de la décision politique, qui mena au déménagement de l'ONF de Toronto à Montréal, me semblait déborder du cadre de ce travail. Ceci dit, mon corpus "épuré" se ramène quand même à une centaine de films.

Afin d'analyser avec exactitude la diffusion de ces films aux États-Unis, il faudrait rappeler tous les facteurs et toutes les politiques qui influencèrent la production au Canada et au Québec. Évidemment, cela excède aussi le cadre de cet article. Aussi, bien que je ponctuerai mon texte d'informations circonstancielles éclairant la présence de films québécois aux États-Unis, je renvoie le lecteur à quelques études plus détaillées sur ce point, celle de Magder (1993) notamment. Par ailleurs, je voudrais rappeler une observation que formulent Bérubé et Magnan (1994) au début de leur étude, à l'effet qu'il est difficile d'obtenir des données complètes sur la distribution dans les salles états-uniennes. Non seulement n'existe-t-il aucune institution chargée de recueillir et de compiler de telles données, mais plus encore, le marché états-unien est d'une telle complexité et d'une telle fragmentation qu'il rend toute compilation extrêmement difficile. D'un côté, il y a les Majors qui distribuent relativement peu de films étrangers, de l'autre, les Indépendants qui s'en attribuent une plus grande part. Mais à cette première découpe s'ajoutent d'autres facteurs de spécialisation: tel Major est doté d'une division "classique" dont l'action se limite à un nombre restreint de salles ou au réseau des collèges et des universités, tel Indépendant se spécialise dans un cinéma de genre, etc.

Le système d'exploitation est lui-même très fragmenté. Des villes comme New York, San Francisco, Los Angeles ou Chicago jouissent d'un statut particulier. On y retrouve plusieurs catégories de salles. Par contre, plusieurs villes, d'un calibre à peine plus petit, figurent sur des circuits plus circonscrits, surtout en ce qui a trait aux débouchés des Indépendants. À notre avis, cette fragmentation ne permet pas au corpus couvert par le $\mathrm{CRC} / \mathrm{R}^{3}$ de refléter ce qui a pu s'écrire dans des journaux régionaux ou d'intérêt local, et qui paraissent dans des villes où des films québécois ont pu être présentés. 
Notre intention est de nous intéresser à tous ces films dans le but de faire le portrait le plus large possible de ce qu'ils sont et de leur réception. Nous diviserons ce vaste corpus (1960-1992) selon des critères d'ordre chronologique et thématique déterminés par l'évolution même de la situation cinématographique au Québec. Nous nous limiterons aussi aux textes qui portent directement sur les films en excluant ceux qui traitent du cinéma québécois en général ${ }^{4}$. Cela fait tout de même beaucoup de textes et beaucoup de films. Mais le portrait de la réception critique est néanmoins biaisé. Aux contingences créées par les systèmes de distribution et d'exploitation auxquels nous avons fait allusion, s'ajoute le fait que plusieurs textes paraissent dans des revues professionnelles - à la suite de visionnements effectués en dehors des États-Unis —, à l'occasion de festivals par exemple, ou dans des salles de projection privées. Il s'agit là effectivement d'une critique américaine, mais cela ne prouve pas que le film a été vu aux États-Uniss 5

\section{Le temps de la découverte: 1960-1972}

Personne ne s'étonnera d'apprendre qu'avant 1960, le cinéma québécois de langue anglaise est très peu diffusé aux États-Unis. Avec le renouveau que connaît le cinéma québécois et canadien dans les années soixante, la curiosité de quelques cinéphiles est cependant alimentée lors de visionnements dans des festivals ou lors de rétrospectives. Si ces cinéphiles n'hésitent pas beaucoup à accorder une place spécifique au cinéma québécois francophone, ils ne concèdent au contraire aucune spécificité au cinéma anglophone qu'ils qualifient de canadien et qu'ils comparent aux films tournés à Toronto ou à Vancouver. Aux yeux de la grande majorité des critiques états-uniens, et cette attitude à notre égard perdure, le dénominateur commun est davantage la langue du film que sa province d'origine ${ }^{6}$. Rares seront les critiques qui trouveront, par exemple, une parenté entre le travail de Don Owen et celui de Claude Jutra.

Lorsque arrivent sur les écrans états-uniens les rares œuvres anglo-québécoises de la période, les points de repère de la critique sur le cinéma canadien sont plutôt minces et renvoient tous à l'ONF. Outre Drylanders (Donald Haldane, 1961), c'est 
Nobody Waved Goodbye (Don Owen, 1964) qui constitue le véritable pôle de référence de ce qu'est le cinéma canadien ${ }^{7}$ : un cinéma à petit budget, réalisé sans vedettes, capable de toucher le cœur du public cinéphile et dont la thématique, tout en s'inscrivant au Canada, réussit à refléter la vie nord-américaine dans son ensemble. On aime la différence, mais à l'intérieur d'une certaine familiarité.

La critique escomptera des films de cet ordre. Et c'est ce qu'elle retrouve partiellement avec Don't Let The Angels Fall (George Kaczender, 1968) et surtout avec Isabel (Paul Almond, 1968). Il faut dire que l'héroöne de ce dernier film, la pétillante et flamboyante "beauté canadienne-française " Geneviève Bujold, n'est pas une inconnue. Elle a joué avec Resnais et De Broca. Cela lui donne des lettres de noblesse au chapitre du cinéma d'auteur.

Isabel is a theory buster. It is an English-language art film; it is an auteur's creation; it is a star vehicle. It is a beautiful and disturbing and exciting film, brilliantly cinematic. And all this for a big fat $\$ 250,000$ in the making. If we want to save face, Hollywood fashion, we can still call it a "foreign" film since it was made in Canada, by Canadians ${ }^{8}$.

Ce suspense cérébral, dont on déplore quelques faiblesses de scénario, possède une âme qui tient autant à Bujold et à Almond qu'aux références explicites aux lieux et à la société québécoise. Bien qu'il soit diffusé par Paramount, d'aucuns s'interrogent sur son éventuel succès. Effectivement, en dépit de critiques enthousiastes, il s'effondrera au box office. Certains le considèrent d'ailleurs comme un des grands "films perdus" de son époque.

A l'inverse, la critique réserve un accueil assez tiède à Prologue (Robin Spry, 1969). Le qualificatif de low budget, s'il n'oblitère pas la dimension auctoriale, se transforme ici en acte d'accusation; il renvoie à "bâclé", "naïf", "simpliste ", voire "ridicule". Seules les situations états-uniennes qu'on y retrouve suscitent un certain intérêt: les manifestations au congrès du Parti démocrate de Chicago et les apparitions éclairs de Ginsberg, Genet et Burroughs sont vues comme l'effort d'un jeune auteur canadien pour pénétrer l'esprit de la jeunesse américaine. C'est pourquoi 
on ne trouve aucun autre avenir à ce film ailleurs que dans les salles d'art et essai et sur les campus.

La déception est plus forte pour High (Larry Kent, 1967). La critique avait gardé un bon souvenir de Caressed (1964), plus proche de son niveau d'attente quant à la nature d'un film canadien. Même si le film obtient un "Film As Art Award " au Festival de San Francisco, la plupart dénoncent une réalisation ennuyeuse, sans imagination, brouillonne et une interprétation amateure. Malgré son allure sérieuse, le film succombe, selon la critique, à la mode érotique, toutes les images de nudité du film le destinant uniquement au marché de la "sexploitation".

En effet, telle est la mode de l'époque. La championne du genre au Québec, la firme Cinépix (où s'activent André Link et John Dunning), vient de réussir la vente de ses trois premiers films à un distributeur états-unien. Deux sont en français, Valérie et L'Initiation (Denis Héroux, 1968 et 1969), le troisième en anglais: Love in a 4 Letter World (John Sone, 1970). Ce dernier s'attire des qualificatifs assez peu flatteurs: réalisé de manière inepte, mal interprété, mis en scène avec ridicule. Variety se demande même si l'objectif de la Société de développement de l'industrie cinématographique canadienne (SDICC) est de produire de tels films et doute qu'on encourage vraiment de cette manière le développement d'un cinéma national ${ }^{9}$. Mais Cinépix rapplique l'année suivante avec un autre film de Sone, Loving and Laughing. Étonnamment, le journaliste de Variety trouvera des vertus à cette comédie érotique qui confronte une commune hippie québécoise avec une famille du Vermont. La "joie de vivre canadienne-française" semble un ingrédient suffisant pour conférer au film une allure nouvelle qui devrait engendrer des dividendes au guichet. Les données à notre disposition ne nous permettent pas de le vérifier.

Malgré tout, en ce début des années soixante-dix, la tendance est plutôt au préjugé favorable envers le cinéma d'auteur. Parmi les Anglo-Québécois qui peuvent prétendre à ce titre, Paul Almond mène le bal. Plusieurs affirment que s'il est en Amérique du Nord un cinéaste anglophone qui mérite cette qualification à cause de l'originalité de son univers et de son écriture cinématographique, c'est bien lui et c'est ce que confirme Act of 
the Heart (1970). Ce film, qui a reçu chez lui une critique dithyrambique, rehausse le prestige du cinéma canadien aux ÉtatsUnis et génère une confiance sincère envers les productions du Canada. Toutefois, malgré la qualité indéniable du jeu de Bujold, malgré l'utilisation fascinante du cadre montréalais, la critique reproche à Almond de s'être attaqué à un scénario à la Dreyer alors qu'il n'a peut-être pas la maturité intellectuelle et humaine nécessaire. Il s'en dégage une sorte de rigidité, pour ne pas dire de frigidité, qui dessert l'œuvre et son propos et l'aligne directement sur le seul marché des salles d'art et essai.

Le film suivant du cinéaste, Journey (1972), malgré l'atout que représente la beauté du paysage de la région de Tadoussac, recevra un accueil plutôt froid; on ne reconnaît plus les intentions du cinéaste dans ce récit symbolique ${ }^{10}$. Mais l'influence d'Almond dans l'entendement qu'a la critique états-unienne de notre cinéma n'est pas à négliger. Ainsi, en voulant expliquer l'intervention gouvernementale canadienne dans la mise sur pied de la SDICC à la fin des années soixante, ce sera en pensant à Act of the Heart qu'un critique écrira :

There was much scoffing and sneering, at the time, because of the traditional Canadian inferiority complex and lack of confidence that competition from the nearby U.S. was too much to expect any kind of meaningful product from Canada; and besides, wasn't the country's film "industry" largely made up of "filmmakers" who wanted to make film of social significance with all those important issues around, and they'd rather work on a shoestring than compromise their product or principle" ?

\section{Le cinéma d'auteur cède sa place}

Si l'étoile d'Almond pâlit quelque peu en ce début des années soixante-dix, d'autres réalisateurs prennent la relève au chapitre du cinéma d'auteur. On y retrouve au premier chef Frank Vitale. Son premier film, Montreal Main (1973), recueille une presse partagée lors de sa présentation au Whitney Museum de New York. On vante l'honnêteté du cinéaste, l'authenticité de son œuvre, son caractère réellement artistique, son habileté à dépeindre des relations humaines transitoires. Le milieu qu'il 
décrit, avec ses connotations homosexuelles, sa moralité assez molle, son penchant pour la drogue, choque ceux qui n'approuvent pas cet univers marginal. Ils sont également déçus par le Montréal de ce milieu, si éloigné des clichés à usage touristique; à leurs yeux, l'absence de pittoresque génère l'ennui. Le film suivant de Vitale, East End Hustle (1976), ne fait que confirmer, aux yeux des New-Yorkais, l'intérêt secondaire du cinéaste.

Par contre, son comédien principal, Allan Moyle, en accédant au statut de réalisateur, ravive la curiosité pour le jeune cinéma anglo-québécois. Si The Rubber Gun (1977) peut irriter de prime abord parce qu'il se situe dans le milieu des petits trafiquants de drogue, il fascine au bout du compte par son côté étrange et brut, par la récupération qu'il fait de l'esthétique du cinéma direct, par son audace formelle et humaine. On évoque à son sujet le cinéma de John Cassavetes, les deux possédant un style analogue où alternent les moments flamboyants et les passages relâchés.

Mais un film attire surtout l'attention sur ce que devrait être le meilleur cinéma anglo-québécois: The Apprenticeship of Duddy Kravitz (Ted Kotcheff, 1974). Meilleur film de l'année aux $26^{\mathrm{c}}$ Canadian Film Awards comme l'avait été en 1971 une réalisation avec laquelle on peut facilement le comparer, Mon oncle Antoine de Claude Jutra, le film obtient l'Ours d'Or à Berlin et la Special Jury Award Gold Medal au Festival d'Atlanta de 1974. Une telle reconnaissance nationale et internationale laisse présager un succès à l'étranger, surtout si le bouche-à-oreille s'avère favorable. La majorité des critiques se montre positive, reconnaissant surtout le jeu des comédiens et la qualité de la réalisation. La minorité déplore le côté confus de l'intrigue et l'ambiguîté des intentions; il faut avouer que peu de critiques semblent connaître Richler et son roman de 1959. Chose certaine, bien que distribué par un Major et bénéficiant, chose rare, d'une seconde sortie un an après sa première, le film ne triplera même pas ses recettes canadiennes. S’il n'a pas été confiné uniquement au marché des salles indépendantes, il n'a pas réellement percé les grands circuits urbains. En fait, le public ne voit pas vraiment de différence avec les films états-uniens qui mettent en scène des types nationaux et The Apprenticeship of Duddy 
Kravitz n'est pas le premier film lui montrant un arriviste juif. D'accord, l'action se passe à Montréal et l'héroïne est canadienne-française, mais le rôle principal est tenu par un Américain, Richard Dreyfuss, auréolé du succès récent d'American Graffiti (George Lucas, 1973). Le film est l'exemple parfait de ce que devrait être, aux yeux de certains, une production canadienne de qualité internationale ${ }^{12}$.

\section{L'industrie se développe}

En mars 1972, Harold Greenberg, président de Bellevue Pathé, annonce la création d'un fonds de plusieurs millions venant compléter l'apport de la SDICC. En l'espace de six mois, cette somme est engagée dans la production de six longs métrages. En mars 1973, Bellevue Pathé fusionne avec Astral. Cette même année, un des patrons d'Astral, Edward Bronfman, se rend à Hollywood avec Greenberg dans le but de mettre sur pied des projets de cofinancement (et non de coproductions). Greenberg déclare à cette occasion :

We want to make Canada a major voice in world entertainment, and the best place to start is in partnership with Hollywood. Since Canada accounts for about ten percent of the domestic market for U.S. films, we feel it is only natural that our country should be actively involved in at least eight to ten percent of the productions made for world consumption ${ }^{13}$.

A partir de ce moment, la majeure partie de la production anglo-québécoise se tourne vers le marché états-unien et les producteurs canadiens montent en épingle le fait d'être distribué làbas. Comme le dit le producteur Pierre David, alors que les distributeurs américains viennent d'acquérir 11 films canadiens: "It's happening. It's time to be cheerfull. This action out of the U. S. for Canadian films is going to separate the men from the boys. Getting U. S. distribution for us is like passing your examination ${ }^{14}$." Tous les films ne bénéficient évidemment pas de cofinancement, mais la très grande majorité est conçue à l'intérieur des paramètres culturels hollywoodiens dans le but de percer ce marché. Dès lors, la presse états-unienne jauge les œuvres à l'aune de son propre cinéma alors que le Québec anglophone 
lui offre majoritairement des films de genre aux allures familières et mettant souvent en vedette des noms qu'elle connaît.

Dans sa volonté d'être semblable, le cinéma anglo-québécois ne craint ni la copie, ni l'emprunt de recettes à succès, au risque de se faire taper sur les doigts. Ainsi, le film le plus ambitieux, The Neptune Factor (Daniel Petrie, 1973), produit par Bellevue Pathé avec la participation de 20th Century-Fox qui avance près de la moitié du budget, s'affuble du sous-titre de "Undersea Odyssey " en référence au célèbre film de Kubrick. Malheureusement, le côté grotesque et absurde du scénario, la faiblesse des dialogues et de comédiens utilisés en dessous de leurs possibilités, ainsi que le piètre talent de Petrie provoquent un accueil des plus sévères. On doute même que de très jeunes enfants puissent éprouver un quelconque plaisir à voir un film qui n'a même pas la prétention d'être un honnête quickie destiné à procurer au moins un peu de détente.

Au contraire, The Pyx (Harvey Hart, 1973) séduit autant Variety que CineFantastique. Ce suspense qui mise à fond sur la performance exceptionnelle de Karen Black et des acteurs de soutien franco-québécois, ainsi que sur l'atmosphère mystérieuse qui se dégage du Montréal superbement capté par René Verzier, apparaît comme le croisement de Rosemary's Baby (Roman Polanski, 1968) avec Klute (Alan Pakula, 1971):

A film of uncommon sensitivity and development particularly for a detective story. It is one of the very few recent films that tries to catch its audience more with character development and sheer professionalism than with brassiness or new tricks. As such it comes as a distinct and rather welcome surprise ${ }^{15}$.

Dans un autre genre, White Line Fever (Jonathan Kaplan, 1975) se mérite aussi une attention particulière. Cette histoire de camionneurs qui se battent contre la corruption sévissant dans leur industrie s'inscrit dans la veine du cinéma d'action "ouvrier" d'apparence progressiste. Le réalisateur, fils du compositeur Sol Kaplan qui figura sur la liste noire de Hollywood, bénéficie d'un préjugé politique favorable. La critique de gauche, celle qu'on retrouve dans Cinéaste et Jump Cut, décortique le film sous toutes ses coutures. Les États-Unis qu'elle y décou- 
vre ne lui font jamais penser qu'elle est là devant un film canadien.

Le Canada (on ne spécifie pour ainsi dire jamais la particularité anglo-québécoise) apparaît sans conteste comme l'arrièrecour du cinéma hollywoodien, ou alors comme un lieu d'apprentissage pour jeunes réalisateurs états-uniens. Le cas le plus célèbre est celui de Seizure (Oliver Stone, 1974). Le futur grand nom de Hollywood vient alors d'obtenir son diplôme de la New York University. Il ambitionne de tourner un film d'horreur de qualité; Greenberg lui donne sa chance. Tous ceux qui apprécient ce genre reconnaissent le succès de son entreprise que l'on qualifie même du plus important film d'horreur depuis The Exorcist (1973). Stone est sacré digne héritier de Peckinpah et de Romero. La mise en image du Québec par Roger Racine reçoit aussi quelques louanges.

\section{David au pays de l'horreur}

L'horreur va occuper une place particulière dans le cinéma anglo-québécois de cette époque et le bénéfice en revient principalement à un seul cinéaste, David Cronenberg. Celui-ci franchit la trentaine quand John Dunning et André Link le recrutent. Cinq de ses films seront produits au Québec, ce qui en fait le plus important cinéaste d'horreur québécois ${ }^{16}$. Son premier film, The Parasite Murders (1975), est, malgré quelques réserves, apprécié par la critique, qui remarque le style du cinéaste, son ironie, son sens de l'exagération, son mélange imaginatif d'humour obscène, de science-fiction et de pornographie. On n'hésite pas à citer à son propos le philosophe Marcuse, qui croit qu'un Éros incontrôlé est aussi fatal que sa contrepartie, l'instinct de mort - ce dont, soit dit en passant, son dernier film Crash (1996) fait encore la preuve. On trouve enfin que si le film d'horreur peut prétendre au titre d'art, le cinéma de Cronenberg en est un bon indice.

The Parasite Murders avait connu une sortie classique pour un premier film de genre: être présenté en programme double. Rabid (1976) bénéficie d'un meilleur sort, car il est distribué par New World Pictures, la compagnie de Roger Corman. On affirme d'ailleurs que Cronenberg est au Canada ce que Corman 
fut pour les États-uniens. La presse quotidienne se montre assez sévère tandis que les amateurs d'horreur, tout à leurs frissons, se délectent de l'atmosphère cauchemardesque dans laquelle le réalisateur baigne Montréal. Cronenberg bénéficie maintenant d'un public. The Brood (1979) confirme ce succès en récoltant, par exemple, $600000 \$$ en 10 jours à Chicago en juin 1979. Même ceux qui n'aiment pas Cronenberg en parlent dorénavant comme du maître canadien de l'horreur et de la peur. Sa mâ̂trise de l'horreur intérieure séduit et ce mélange de beauté et de laideur produit un effet cathartique qui approcherait des réussites d'Ingmar Bergman.

Jamais un cinéaste canadien n'avait tant percé sur le marché états-unien. Ainsi Scanners (1980) bénéficiera d'une sortie dans plus de 450 salles. Souvent la critique mentionnera l'origine du cinéaste ou du film sans que cela ne renvoie à une quelconque connotation négative. On rappelle même que Cronenberg préfere travailler à sa manière au Canada plutôt que de s'expatrier aux États-Unis. Scanners partage encore une fois la critique car, de tous les genres, ce sont peut-être l'horreur et la science-fiction qui ont le plus de difficulté à être prises au sérieux. Néanmoins, ses admirateurs rangent Cronenberg au même niveau que les réalisateurs Brian De Palma, John Carpenter, George Romero et le romancier Stephen King. Les producteurs Pierre David et Claude Héroux doivent se réjouir d'une telle reconnaissance.

Avec Videodrome (1982), Cronenberg réussit à obtenir un financement de Hollywood. Il s'estime par ailleurs prêt à signer une œuvre plus personnelle, à mériter son statut d'auteur. Il produit un des films les plus originaux réalisés dans sa catégorie. Cette étonnante et superbe odyssée du corps et de l'esprit, de l'homme et de la machine, cette réflexion philosophique et sociale sur le pouvoir des médias de masse déroutera malheureusement le public moyen. Tout en reconnaissant que Cronenberg est un génie dans son genre, certains critiques trouveront qu'il a surestimé cette fois-ci son public.

Le cinéma francophone ne semble pas du tout inspiré par le succès de Cronenberg, mais ce n'est pas le cas des anglophones, qui ne craignent d'ailleurs pas de copier des succès récents. Durant les années quatre-vingt, la production anglo-québécoise 


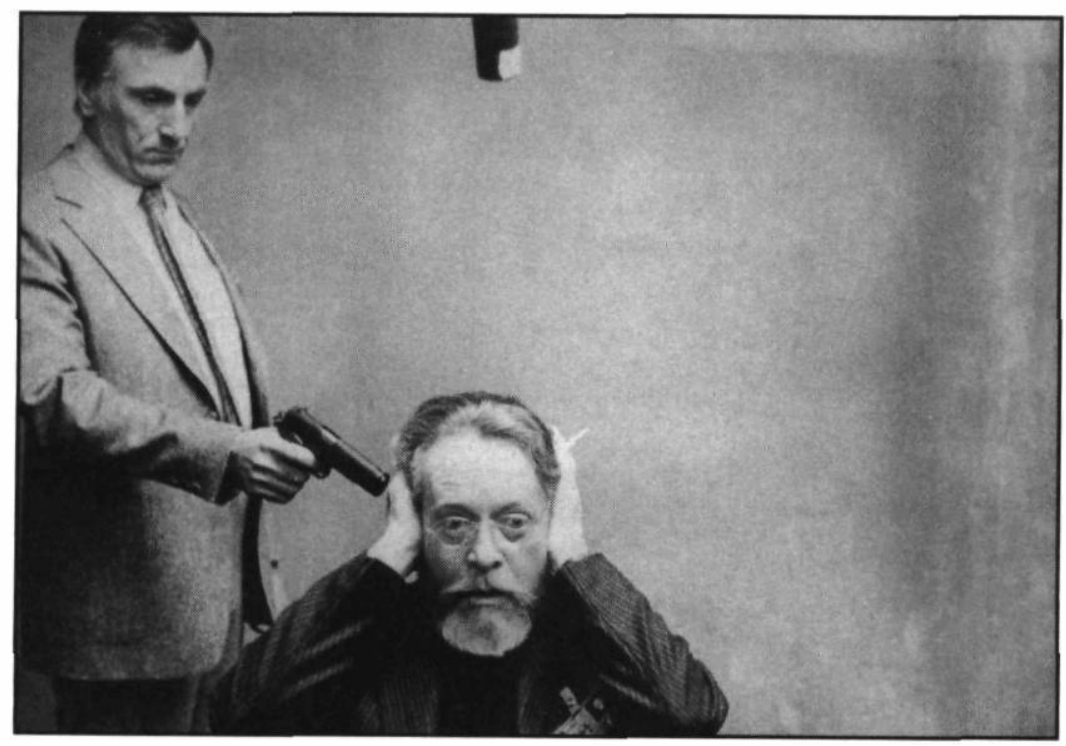

\section{Scanners de David Cronenberg (1980)}

Collection Cinémathèque québécoise

se précipite littéralement sur le film d'horreur et de peur qui autorise, on le sait, l'existence de films à budgets réduits. Pensons à My Bloody Valentine (George Mihalka, 1980) qui s'attire le curieux compliment de n'être pas le pire des douzaines de gorenographic movies à petit budget à envahir les écrans. Voilà ce qui explique sans doute qu'il soit distribué par la Paramount et non par une compagnie de moindre importance comme c'est le cas habituellement. L'atmosphère qui se dégage du décor et de l'univers minier joue aussi en faveur du film.

Tel n'est pas le cas de Death Ship (Alvin Rakoff, 1980), une coproduction avec la Grande-Bretagne. Il s'attire les quolibets les plus sévères: une nullité repoussante, une réalisation amateure, sans inspiration, des situations absurdes. Les comédiens connus qui participent à une telle entreprise se dévalorisent. Même CineFantastique, d'ordinaire sympathique à l'horreur, trouve que le film se fourvoie. Cette production de Bellevue Pathé, où seul René Verzier s'attire quelques compliments, répondrait à peine aux attentes des ciné-parcs et pourrait, à la rigueur, servir de deuxième partie à un programme double ou de 
film en fin de nuit à la télévision. Le Canada lui-même s'en tire plutôt mal, ainsi que l'indique un critique: "It's a totally ludicrous effect - produced with the participation of the CFDC - in which the most horrifying aspect is the fact that the film has been made ${ }^{17}$."

Malheureusement, un tel son de cloche revient à moultes reprises tant la médiocrité de la production canadienne hérisse plusieurs observateurs états-uniens. Ainsi lit-on à propos de Happy Birthday to Me (J. Lee Thompson, 1980) : "Obviously an inept Canadian copy of the earlier American horror successes, this film arrives much too late to be anything but a weak and confused parody of the precedents ${ }^{18}$." Inversement, on trouvera que Of Unknown Origin (George P. Cosmatos, 1983), une histoire de rats qui se déroule à New York, ne possède pas cette foisci le petit côté minable des productions canadiennes à petit budget et que son récit apparaît bien ficelé. Peut-on se consoler en pensant que notre cinéma n'est pas pire que bien des films étatsuniens? Pour plusieurs journalistes, ces films ne sont que des sous-produits mal fagotés, des produits pour adolescents attardés. On tergiverse face à Spacehunter: Adventures in the Forbidden Zone (Lamont Johnson, 1983), une production qui a coûté plus de 13 millions ${ }^{19}$, dont le seul intérêt est de tenter de redonner vie au cinéma en relief et d'y adjoindre du son en stéréoDolby. On méprise Zombie Nightmare (Jack Bravman, 1986), tellement mauvais sous tous ses aspects qu'on lui épargnera le supplice de la salle pour le dériver vers la vidéocassette. Tout comme The Kiss (Pen Densham, 1988), où n'émerge aucune idée, ou Cursed (Mychel Arsenault, 1990), pourri à un degré qui dépasse l'imagination. L'analyste canadien ne peut manquer d'éprouver une certaine honte à lire tous ces textes où canadien veut dire pitoyable, insensé, répugnant, d'intelligence réduite. Est-ce là les produits valorisés par David, Solnicki, Link, Dunning, Claude Héroux et Greenberg? Ne peuvent-ils faire la différence entre un Cronenberg et des fonds de poubelle ${ }^{20}$ ? Heureusement que le public états-unien friand de films d'horreur y voit un peu clair et déserte ces œuvres qui se moquent de lui.

En fait, sur une douzaine d'années, seuls quelques films reçoivent un accueil qui n'est pas totalement négatif. Ainsi, Terror 
Train (Roger Spottiswoode, 1979), un ersatz de Halloween (John Carpenter, 1978) produit par Greenberg avec de l'argent hollywoodien, bénéficie d'une réception mitigée où la qualité technique de l'ensemble et certaines surprises au niveau du scénario semblent élever cette réalisation au-dessus des œuvres qui font partie du film d'horreur. De son côté, Pin (Sandor Stern, 1988) semble davantage jouer la carte de la psychologie au détriment de l'horreur, ce qui lui vaut plusieurs notes d'appréciation. Whispers (Douglas Jackson, 1990) surprend même par sa nouveauté et par la bonne performance de Jean Leclerc et de Jackie Burroughs; mais ce film est destiné uniquement au marché de la vidéo.

\section{Le cas Jean-Claude Lord}

La grande majorité des films que nous venons de mentionner a été réalisée par des Anglo-Québécois, des Canadiens anglais, des étrangers, mais rarement par des Franco-Québécois. C'est ici que le cas de Jean-Claude Lord s'avère intéressant. Pendant 15 ans, son nom avait été associé au cinéma commercial francophone auquel le public réservait un accueil des plus corrects. En 1980, il décide de se réorienter vers la production en anglais. Il explore toutes les avenues qui s'ouvrent à lui. Lorsqu'il débute, les films de peur et d'horreur semblent la meilleure porte pour pénétrer le marché états-unien. Lord va l'emprunter. D’après les informations disponibles, Visiting Hours (1981) s'en tire bien quoique tardivement au box office. En effet, il ne sort que quelques années après sa production grâce à une entente entre 20th Century-Fox et le producteur québécois Filmplan International. La presse, même celle vendue au cinéma de genre, démolira le film. Lord et son opérateur Verzier passent pour des amateurs. On dénonce la minceur et la vacuité d'un récit qui ne recherche que l'effet choquant où on semble prendre plaisir à montrer les victimes se faire tuer par un quelconque détraqué. Ce premier film de Lord en anglais vient renforcer l'idée qu'ont les Américains de ce que sont généralement les films canadiens produits grâce à des mesures fiscales et destinés à leur marché: ennuyeux, lourds, pleins de clichés.

Les films subséquents de Lord bénéficient d'un sort encore pire. Leur diffusion semble parcellaire, limitée à des sorties 
régionales après avoir dormi quelque temps sur les tablettes. Le destin de ces films pose d'ailleurs clairement la question du marché des productions anglo-québécoises et en montre les limites. Les sujets couverts par le réalisateur indiquent bien le caractère opportuniste de ces productions qui se dirigent au hasard vers des objectifs mal définis. Après avoir poussé une pointe du côté de la mode et raccolé le spectateur en manque de chair dénudée (Covergirl, 1982), Lord tente, dans The Vindicator (1984), de mélanger horreur et science-fiction dans un récit aux circonvolutions telles qu'on prendra le film pour une parodie de l'horreur. Effectuant un virage à 180 degrés, il s'attaque ensuite au film pour la famille avec Toby McTeague (1985). Parce que cette histoire de course de chiens se déroule dans le Grand Nord québécois, certains y verront une volonté de renforcement de l'identité canadienne. Même canadianisation avec Mind Field (1989) qui traite du scandale des expériences sur le LSD menées par la CIA à Montréal; même accueil critique où produit canadien signifie pauvreté des sujets et des moyens ${ }^{21}$.

Laventure anglophone de Lord s'arrête avec Eddie and the Cruisers II: Eddie Lives (1989), un sequel d'un film de rock and roll purement états-unien mis à la sauce canadienne, un mariage réussi aux yeux de quelques-uns: "Unusual among recent Canadian productions aimed at general U. S. audiences, pic refreshingly doesn't hide its north-of-the-border content, with frequent emphasis on the Montreal local (including snowy scenes and hockey games) ${ }^{22}{ }^{2}$. Cette fois-ci, le métier de Lord semble s'imposer, surtout dans les suites musicales auxquelles il impose un rythme dynamique que la presse apprécie.

\section{Retour aux genres}

Tout comme chez Lord, le cinéma anglo-québécois de genre ne se limite pas à l'horreur et au fantastique, bien que ceux-ci dominent. Il va dans toutes les directions. Ainsi dans la catégorie du film pour la famille que Lord a déjà explorée, il faut mentionner qu'on retrouve, outre Jacob Two Meets the Hooded Fang (Theodore J. Flicker, 1977), quatre "Contes pour tous", The Peanut Butter Solution (Michael Rubbo, 1985), bien reçu au Festival du film pour enfants de Chicago, The Great Land of 
Small (Vojtech Jasny, 1987), Tommy Tricker and the Stamp Traveller (Michael Rubbo, 1987) et Vincent and Me (Michael Rubbo, 1990). Ces films bénéficient d'échos positifs bien qu'on souligne leur rythme lent et la manière dont ils different d'avec les pratiques états-uniennes en ce domaine. On croit que leurs charmes devraient leur ouvrir les portes de la télévision et de la vidéocassette.

Le drame historique va aussi tenter les producteurs. L'accueil réservé à Two Solitudes (Lionel Chetwynd, 1977) s'avère favorable; celui que reçoit The Lucky Star (Max Fischer, 1980) est plus mitigé. Cette histoire de Juifs hollandais qui se déroule durant la Seconde Guerre mondiale apparaît bien improbable, mais elle séduit, elle remue les cœurs et se mérite les louanges de ceux qui ne croyaient plus en la capacité des films "abris fiscaux" de n'être autre chose que des navets. Une autre histoire de guerre, qui se déroule en 1914 celle-là, The Wars (Robin Phillips, 1982), touche peu la presse, tout comme Bethune: The Making of a Hero (Philip Borsos, 1990). Ces films manquent d'action, de rythme, de passion et ne paraissent passables que comme séries télévisées. Les mêmes reproches s'adressent à la coproduction canado-francoisraëlienne Eminent Domain (John Irvin, 1990) dont l'action, tournée en Pologne, est censée se dérouler à l'intérieur du politburo polonais au temps de la guerre froide. Avec ces drames historiques, le cinéma anglo-québécois tire à peine ses marrons du feu.

Le rire est, dit-on souvent, ce qui s'exporte le moins bien, car chaque population a ses propres critères et ses propres références. En ce domaine, le cinéma et la télévision aux États-Unis ont toujours su être en phase avec leur public. Cela a-t-il constitué un frein pour le cinéma anglo-québécois? À y regarder de près, rares sont ceux qui se sont aventurés dans la comédie, et cela même au Canada anglais. La première comédie angloquébécoise qui arrive sur le marché américain est Dirty Tricks (Alvin Rakoff, 1980). Censé se dérouler à Boston, ce film à poursuites ne fait pas rire, sauf pour de mauvaises raisons. The Funny Farm (Ron Clark, 1981) s'en tire un peu mieux, car cette comédie dramatique apparaît au moins comme une vitrine convenable pour plusieurs comiques canadiens, même si on ne lui prédit aucun avenir sur le marché des États-Unis. 
En fait, à cette époque, il n'y a qu'une seule exception à la règle, et elle est de taille: Porky's (Bob Clark, 1981). Ce film est québécois par défaut, car ce scénario a été proposé par des ÉtatsUniens à Harold Greenberg pour diminuer les risques et bénéficier des mesures fiscales canadiennes ${ }^{23}$. La part de Greenberg s'élève seulement à $37 \%$ du budget. La presse corporative prédit l'échec du film mais des tests préalables en région prouvent qu'il rejoint son public. En fait, au plan commercial, ce sera le plus important succès de tous les temps ${ }^{24}$. En dépit ou peut-être à cause de sa vulgarité, de son mauvais goût, d'un scénario anémique, il est devenu un film-culte. Mais les deux sequels, Porky's II: The Next Day (Bob Clark, 1983) et Porky's Revenge (James Komack, 1985) ne connaîtront jamais un tel succès. La sauce s'étire et s'affadit, l'effet de surprise n'est plus là, les gags se laissent deviner tant ils sont bêtes.

On tentera aussi d'exploiter le succès de la série télévisée SCTV où triomphe John Candy. Mais Going Berserk (David Steinberg, 1983), produit par Claude Héroux et Pierre David avec cofinancement états-unien, sera descendu en flammes comme l'exemple même de la comédie inepte, mortellement ennuyeuse et totalement non professionnelle, et cela malgré la présence de vedettes très connues ${ }^{25}$. Interprété par le duo Elliott Gould/Kate Jackson, Dirty Tricks (Alvin Rakoff, 1980) passera pour une comédie manquée, fabriquée par des gens qui ne savent pas pourquoi les films ont du succès, ni comment faire rire. En outre, son sujet "américano-patriotique" bostonnais semble biscornu quand on sait que le film provient du Canada. Les critiques états-uniens n'ont aucune difficulté à apprécier les films de genre en tant que tels et c'est sous cette lumière qu'ils jaugent ces comédies anglo-québécoises. Cela finira par convaincre les producteurs québécois qu'ils ne peuvent concurrencer les États-Uniens sur ce terrain ${ }^{26}$.

Le Québec possède une autre tradition depuis Valérie: celle du film érotique. Il n'est pas étonnant que Cinépix, la compagnie qui a produit ce film et plusieurs autres du même ordre, tente sa chance en langue anglaise. Le résultat sera des plus déplorables. Ainsi la série des Ilsa se range dans la catégorie des pires soft-porn, qui mélangent sexe et violence sadique ${ }^{27}$, alors 
que The Surrogate (Don Carmody, 1984), qui mise sur la beauté de Carole Laure, manque totalement d'attrait et de lustre. De son côté, la compagnie RSL, dirigée par Robert Lantos et Stephen Roth, n'hésite pas à exploiter les sujets les plus débiles dans l'unique but de mettre à l'écran quelques corps féminins. La presse les situe au niveau intellectuel de préadolescents qu'exciterait la littérature rose. Qu'ils présentent un groupe de filles qui veut s'envoyer en l'air avec des joueurs de hockey (One Night Only, Timothy Bond, 1984) ou qu'ils nous entraînent du côté de la danse-exercice (Heavenly Bodies, Lawrence Dane, $\left.1984^{28}\right)$, ces films n'ont d'intérêt que pour les voyeurs qui dorment au fond de certains cinéphiles. Tous ces films s'attirent un souverain mépris de la part de la critique. Elle dénonce leur amateurisme et leur vulgarité, déplore le profond ennui qu'ils engendrent, ironise sur leur imaginaire de guimauve, trouve même qu'il s'agit là d'exemples gênants de ce que les gouvernements au nord de la frontière appellent avec euphémisme le contenu canadien. Il n'est pas étonnant que les récriminations du Canada quant à la protection de sa culture ne pèsent pas lourd aux yeux de plusieurs États-Uniens dans la mesure où ils ne voient, en provenance de ce pays, que des sous-produits de leur propre culture ${ }^{29}$.

\section{Un cinéma du semblable bien à l'abri... fiscal}

Comme on le constate, pratiquement tous les genres sont couverts par les firmes québécoises, car l'objectif est de jouer dans les mêmes ligues, quoique mineures, que les Américains ${ }^{30}$. C'est d'ailleurs à l'intérieur de ces paramètres que le cofinancement s'applique le plus. Un exemple parmi d'autres: l'association d'International Cinemedia Center avec la Columbia, dans le but de produire au Canada trois à six films par année dont les budgets respectifs seraient de moins de deux millions $\$^{31}$. Dans ce contexte, il ne faut pas s'étonner de voir le Québec travesti si souvent en lieux de tournage étrangers que de le reconnaître devient presque un petit jeu du genre "Cherchez l'erreur». Outre les exemples que nous avons signalés précédemment, mentionnons Breaking Point (Bob Clark, 1976), censé se dérouler à Philadelphie, Blackout (Eddy Matalon, 1978) à New York, Hard Feelings 
(Daryl Duke, 1981) à Long Island en 1963, etc. ${ }^{32}$. Règle générale, la critique n'apprécie pas cette supercherie ou alors en rit; la critique préfere qu'une production canadienne visant le marché états-unien soit tournée aux États-Unis ${ }^{33}$.

Une autre stratégie utilisée par les producteurs québécois pour percer le marché états-unien consiste à suivre les modes et plagier les succès. Nous l'avons suggéré quand nous avons parlé de The Neptune Factor. Avec City On Fire (Alvin Rakoff, 1978), l'intention est encore plus manifeste. On s'engouffre directement dans la vogue du film-catastrophe lancée par The Towering Inferno (Irwin Allen, John Guillermin, 1974), en misant sur des comédiens connus comme Henry Fonda et Ava Gardner. Mais encore une fois, malgré un budget au-dessus de la moyenne ${ }^{34}$, la faiblesse du scénario bousille tout; des personnages mal construits, des péripéties ennuyeuses montées sans rythme, un récit mal structuré, et cela sans parler des effets spéciaux à moitié réussis, voilà ce que la presse reproche au film. Un journaliste observe même que dans le genre du film-catastrophe, les Japonais réussissent beaucoup mieux, rappelant du même coup que le Canada n'est pas le seul pays à tenter de percer sur le marché états-unien en imitant leur production de série $\mathrm{B}$.

L'accueil est encore plus sévère pour Paradise (Stuart Gillard, 1981). La critique trouve embarrassant que le film copie si explicitement tant de films pour n'être en définitive qu'un sousBlue Lagoon (Randal Kleiser, 1980). On estime que faire un mauvais film est une chose et en est une autre le fait de s'approprier sans vergogne le travail d'autrui et de le gâcher. On décèle là le genre d'anémie intellectuelle et le manque d'imagination qui ont caractérisé jusqu'alors, aux yeux de plusieurs, l'association du distributeur américain Avco-Embassy avec la compagnie montréalaise RSL. Et la situation ne s'améliore pas lorsqu'on regarde le sort réservé à Speed Zone (Jim Drake, 1989), un ersatz de Cannonball Run II (Hal Needham, 1984) portant sur la course automobile. Seul John Candy semble s'en tirer honorablement.

Il arrive parfois qu'un film canadien devance les États-Uniens dans le choix du sujet, mais qu'il sorte en salles là-bas quelques années plus tard, lorsque sa parenté avec un succès états-unien 
peut laisser espérer une chance commerciale. One Man (Robin Spry, 1977), une production de l'ONF qui avait été présentée au Los Angeles Filmex de 1978, constitue un exemple de ce phénomène. La parenté qu'il présente avec The China Syndrome (J. Bridges, 1979) ainsi que le succès de Len Cariou à Broadway expliquent sa sortie tardive en 1979 . La presse se montre mitigée, mais le style de Spry ne déçoit pas. Dans les circonstances, on estime que le meilleur marché de ce film sera la télévision.

Le même facteur joue aussi dans le cas des vedettes. Prenons le cas de l'États-Unienne Jodie Foster dont la carrière, jusqu'en 1974 , se déroule plutôt à la télévision. Alors qu'elle est relativement inconnue, on la retrouve dans deux films québécois, Echoes of A Summer (Don Taylor, 1975) et The Little Girl Who Lives Down the Lane (Nicholas Gessner, 1976 ${ }^{35}$ ). Dans le premier cas, la jeune Foster dame radicalement le pion à son partenaire, le vétéran Richard Harris. Dans le second, on la qualifie de comédienne extraordinaire dans son rôle de jeune meurtrière. Il faut dire que ce dernier film, tourné avant Taxi Driver (Martin Scorsese, 1976), n'est distribué aux États-Unis qu'après celui-ci, au moment où la valeur commerciale de Jodie Foster a augmenté.

Les productions anglo-québécoises destinées au marché étatsunien jouent aussi, autant qu'elles le peuvent, les cartes du vedettariat états-unien et international. Il suffit de penser à Sophia Loren et John Huston dans Angela (Boris Segal, 1977), à Robert Mitchum et Lee Majors dans Agency (George Kaczender, 1979), à Elliott Gould et Kate Jackson dans Dirty Tricks (Alvin Rakoff, 1980) ou encore à Rod Steiger dans The Lucky Star (Max Fischer, $1980^{36}$ ). Le fait que quelques vedettes soient connues aux États-Unis permet d'atteindre l'objectif commercial du name value tout en répondant aux exigences gouvernementales canadiennes qui obligent à ce qu'un certain nombre de postes soient occupés par des Canadiens pour que le film puisse bénéficier de juteux avantages fiscaux. Cela explique la présence ultrafréquente de Donald Sutherland ${ }^{37}$, de John Candy ${ }^{38}$, d'Alexandra Stewart ${ }^{39}$ ou encore celle de Michael Sarrazin ${ }^{40}$, sans parler de l'ineffable Margaret Trudeau, "vedette" de Kings and Desperate Men (Alexis Kanner, 1981). 


\section{L'éternel salut par l'étranger}

Tous ces facteurs se combinent et s'amplifient lorsqu'on s'engage sur le terrain de la coproduction. Aux yeux d'une partie de la profession, l'expansion de la production québécoise et canadienne reposerait sur la collaboration avec l'étranger et les différents directeurs de Téléfilm ou de la Sodec ne manquent pas les occasions de répandre cette "vérité". Dès le milieu des années soixante-dix, les producteurs québécois entrent de plain-pied dans cette aventure, surtout les frères Claude et Denis Héroux. Nous avons évoqué plusieurs fois le cofinancement canado-américain. Nous parlerons plutôt maintenant des coproductions. La France y occupe une place de choix mais, paradoxalement, plusieurs des coproductions franco-canadiennes se tournent en anglais, cherchant ainsi à s'internationaliser plus aisément ${ }^{41}$. Cette pratique n'est pas uniquement le fait de Français. Ainsi Denis Héroux tourne en France Jacques Brel is Alive and Well and Living in Paris (1974), ce qui lui vaut de passer aux ÉtatsUnis pour un "young French director" tandis que Brel sera qualifié de "little-known Belgium composer». Malgré un cadre de sortie prestigieux - sur abonnement à l'American Film Theatre - , la presse lèvera le nez sur ce musical de série B, bourré de clichés et de sentimentalisme.

Évidemment, ce partenariat ne s'applique pas de manière égale dans tous les domaines. Du côté des comédiens, ce sont principalement les étrangers qui s'imposeront alors que les Québécois seront confinés aux boulots techniques où ils excellent souvent d'ailleurs et dont la critique reconnaît le talent, bien avant la critique québécoise qui tourne tellement le dos à tous ces films, qu'elle n'y voit généralement aucun aspect positif. Autant, sinon plus, que les autres films de notre corpus, ces films internationaux ne possèdent aucune saveur canadienne et la majorité vogue dans les basses eaux de la médiocrité.

Il est assez aisé de remarquer que le fait d'être tourné en anglais ne garantit pas une distribution commerciale de premier ordre; ce fait à lui seul démontre l'absurdité des coproductions franco-canadiennes tournées en anglais. Des films comme Cathy's Curse (Eddy Matalon, 1976), Blackout (Eddy Matalon, 1978), It Rained All Night The Day I Left (Nicholas Gessner, 
$1978^{42}$ ), Black Mirror (Pierre-Alain Jolivet, 1980), Joy (Serge Bergon, $1983^{43}$ ), et Money (Steven H. Stern, 1991) sont à peine diffusés aux États-Unis et obtiennent une presse défavorable, quand ils en ont une... On trouve tout de même quelques cas curieux ${ }^{44}$. Mentionnons-en deux: Honeymoon (Patrick Jamain, 1985), une sorte de "Un Français à New York" que la presse new-yorkaise trouve plutôt morne et mal interprété par Nathalie Baye et Richard Berry; et surtout Candy Mountain (Robert Frank \& Rudy Wurlitzer, $1987^{45}$ ).

Mais au moins quelques films surnagent aux yeux des ÉtatsUniens. The Little Girl Who Lives Down the Lane (Nicholas Gessner, 1976) procure quelque plaisir grâce au talent de Jodie Foster et à la beauté des images de René Verzier; celui-ci donne à la région de Knowlton une allure de Nouvelle-Angleterre tout à fait poétique qui compense les carences du scénario. Full Circle (Richard Loncraine, 1977) obtient, quant à lui, le Grand Prix au Festival du cinéma fantastique d'Avoriaz, une récompense bienvenue pour ce petit film d'épouvante bien construit et bien interprété par Mia Farrow.

Avec Atlantic City, U.S.A. (Louis Malle, 1980), la critique jubile véritablement: Burt Lancaster donne un numéro d'une finesse qu'on ne lui avait vue depuis longtemps, Susan Sarandon est drôle et touchante et le film, grâce au talent de Malle - qui n'en est pas à ses premières armes états-uniennes - et de son scénariste John Guare, nage dans l'élégie et la poésie. Si jamais une coproduction franco-canadienne put jamais prétendre atteindre le haut de gamme, c'est bien ce film. Comme le dit un critique, et il n'est pas le seul à le penser, "[...] so far the best film to come from north of the border ${ }^{46} "$. Mais en même temps, la majorité des critiques considère ce film comme étatsunien, même celui qui écrit :

\footnotetext{
Curiously, the fact that Atlantic City was a CanadianFrench co-production seems to work in its favor as a perceptive glimpse of a community in architectural and spiritual transition. Canadian producer Denis Héroux and Canadian cinematographer Richard Ciupka seem to have brought an anthropological-sociological curiosity to bear on the subject ${ }^{47} \ldots$
} 
C'est pourquoi un sondage auprès des critiques états-uniens (paru dans la revue American Film, le 2 novembre 1989) place le film au cinquième rang des 20 meilleurs films états-uniens des années quatre-vingt. Personne ne le situe parmi les films étrangers $^{48}$. La consécration lui viendra à Venise, où il obtient le Grand Prix du Festival en 1980. Encouragé par ce triomphe, le duo Denis Héroux-John Kemeny récidive avec Quest for Fire (Jean-Jacques Annaud, $1981^{49}$ ). Dans l'ensemble, la presse, globalement séduite par cette odyssée préhistorique, vante la beauté des images et les paysages grandioses, le travail sur le langage et la perfection des maquillages. On lui reproche cependant souvent son racisme et son sexisme, qui glorifient la supériorité de certains sur d'autres.

La presse se montre également attentive aux films réalisés par des réalisateurs renommés. Ainsi Claude Chabrol, qui n'en est pas à ses premières armes en anglais et dont plusieurs connaissent le travail, pique la curiosité des critiques avec The Blood of the Others (1984), d'autant plus que son film adapte un roman de Simone de Beauvoir qui se déroule en France sous l'Occupation. Philippe de Broca jouit d'une notoriété moindre et c'est plutôt un réalisateur sur le déclin qu'engage Héroux pour tourner Louisiana (1984), une coproduction à grand spectacle qui fait trois heures pour le cinéma et six pour la télé. D'aucuns notent que le film passe mal au grand écran et qu'il n'aurait jamais dû y aboutir. Ce sera directement au marché de la vidéocassette que s'adressera Double Identity (Yves Boisset, 1990). Sans toujours savoir que Boisset est identifié chez lui à la tradition hollywoodienne, la critique trouvera le récit bien mené et les comédiens européens et canadiens dirigés avec talent. Finalement Shadow of the Wolf (Jacques Dorfmann, 1992), malgré les moyens dont il dispose, a de la difficulté à convaincre, on lui trouve un côté condescendant avec les Inuits et on croit qu'il s'adresse en définitive aux enfants en manque d'exotisme.

Les Français ne sont pas les seuls étrangers à tourner en anglais au Québec; les Britanniques y viennent aussi. La presse états-unienne ne débordera pas d'enthousiasme pour les quelques films qu'ils réalisent. Ainsi, malgré la présence d'Oliver Reed et de Donald Pleasence, Tomorrow Never Comes (Peter 
Collinson, 1977) est perçu comme un mélodrame sans personnalité où foisonnent les clichés. Par ailleurs, la plupart des critiques trouvent que The Hotel New Hampshire (1984), cofinancé avec les États-Unis mais tourné par l'Anglais Tony Richardson, arrive bien en dessous du roman de John Irving (bien qu'un universitaire estime qu'il s'agit là d'une des adaptations les plus fidèles qu'il connaisse et un des meilleurs exemples du genre ${ }^{50}$ ). On ne trouve à ce film que quelques qualités au plan de la distribution (américaine) et des paysages québécois qui évoquent si bien la Nouvelle-Angleterre. Tous s'entendent cependant pour dire qu'il s'agit du meilleur Richardson depuis Tom Jones (1963), une manière d'affirmer que ce cinéaste sur le retour, versé depuis longtemps dans l'académisme, était le seul que le Canada pouvait s'offrir.

\section{Un cinéma plus personnel}

Mais il arrive que les étrangers réalisent des œuvres qui témoignent de plus de personnalité. Ainsi le Tchèque Jan Kadar décrit avec nostalgie, dans Lies My Father Told Me (1974), le milieu juif montréalais des années vingt. La presse rapproche le film de Hester Street (Joan Micklin Silver, 1975), sorti aux États-Unis avant le film de Kadar. Quelques-uns le trouvent trop mélodramatique, bourré de clichés sentimentaux. Mais la majorité aime son humanisme, son romantisme, la douceur de sa musique. C'est ce qui explique qu'il ait obtenu, aux Golden Globes de février 1976, le prix du meilleur film en langue étrangère ${ }^{51}$. Même le singulier Werner Herzog fait partie de ce courant; Scream of Stone (1991), coproduit avec l'Allemagne et la France, parait toutefois fort obscur et un peu fou, ce qui lui coupe tout marché commercial.

Malgré une tendance internationaliste et commerciale qui engendre beaucoup de mauvais films, plusieurs cinéastes canadiens ont également la prétention de réaliser un cinéma personnel, qu'il appartienne ou non à un genre ${ }^{52}$. Nous avions signalé quelques cas pour les années soixante. Dans les décennies ultérieures, leur nombre augmente. Prenons comme exemple In Praise of Older Women (George Kaczender, 1977), qui est perçu par plusieurs comme un film sympathique glorifiant les 
prouesses amoureuses des femmes d'âge mûr et accordant un soin particulier à la beauté des images. Ses détracteurs y voient plutôt un softcore qui se donne une allure de grande classe. A leurs yeux, la référence à la Révolution hongroise de 1956 constitue la vraie dimension pornographique du film en ce qu'elle banalise la tragédie d'un peuple ${ }^{53}$.

Robin Spry avait attiré quelques regards à la fin des années soixante. En 1977, il réalise à l'ONF un premier long métrage de fiction, One Man, dont nous avons précédemment situé le contexte de sortie états-unien. Ce thriller politique à connotations sociales situait toujours le cinéaste dans la ligne des auteurs engagés. Lorsqu'il quitte l'ONF après ce film, son cinéma hésitera entre la description du milieu politique et culturel québécois et la recherche d'un cinéma commercial pour grand public. La presse américaine se montre souvent sévère face à cette évolution. Ainsi Suzanne (1980), dont le sujet paraît ambitieux et original, est perçu dans l'ensemble comme un désastre qui fait rire alors qu'il ne le devrait pas. Par contre Keeping Track (1986), qui met en vedette Michael Sarrazin et Margot Kidder et qui se déroule dans le milieu de la télévision montréalais, semble soutenir la comparaison avec les films analogues sortis aux États-Unis. Enfin Obsessed (1988) captive l'intérêt de la presse d'abord par son côté modeste et maîtrisé, ensuite par son sujet, un délit de fuite commis par un États-Unien de passage à Montréal. On apprécie surtout que cela serve de prétexte à l'auteur pour traiter avec subtilité des relations d'amour-haine entre les États-Uniens et les Canadiens.

Ted Kotcheff fait aussi partie de ces auteurs dont la carrière débute avant le boom international. Son cas est plus particulier dans la mesure où il va s'établir à Hollywood en 1974 pour y poursuivre son travail. Son intérêt pour Richler et l'impossibilité de monter un tel film aux États-Unis explique son retour au Québec. Malheureusement, Joshua Then and Now (1985) ne bénéficiera pas d'un aussi bon accueil que Duddy Kravitz, car aux yeux des États-Uniens, le héros y est moins sympathique, surtout dans la partie contemporaine de la diégèse. La beauté et la sauvagerie des paysages ne compense pas la faiblesse du scénario. Bien qu'il puisse s'en tirer sur le marché d'art et d'essai, il s'agit d'un échec, car il visait le grand public. 
Claude Gagnon, un des rares Québécois francophones avec Jean-Claude Lord à tourner fréquemment en anglais ${ }^{54}$, vise aussi à toucher le grand public dans le cadre d'une démarche originale. The Kid Brother (1987), qu'il tourne à Pittsburg, obtient une presse positive à sa sortie; on lui prédit un succès aux ÉtatsUnis. Malheureusement, dans les journaux recensés, on retrouve peu d'échos de sa carrière ultérieure nous permettant d'évaluer son impact. The Pianist (1991) est reçu avec beaucoup de réserves et, au mieux, lui prédit-on une carrière à la télévision. Les tentatives de Gagnon de réaliser un cinéma commercial anglophone d'envergure internationale semblent faire long feu.

Il existe cependant des Anglo-Québécois dont les prétentions auctoriales sont affirmées avec plus de clarté. Il convient de mentionner Horses in Winter (Rick Raxlen, 1988), dont la facture très personnelle séduit et deux films de Mort Ransen, Bayo (1984) et Falling Over Backwards (1990), dont on admet les qualités au plan du scénario et du jeu. Mais le cas le plus curieux demeure celui de Bachar Chbib. On reconnaît à Memoirs (1984) un style punk perverti qui justifie sa présentation à New York dans la catégorie des "films de minuit ". Avec Evixion (1986), les choses se gâtent. On ne sait comment aborder ce film qui mélange l'expérimental et la caricature, le tout dans une sorte de soupe narrative incohérente qui reste sur l'estomac. Un journaliste trouve même qu'il s'agit là d'un bon exemple du gaspillage de l'argent des contribuables ${ }^{55}$. Étant donné sa volonté de percer aux États-Unis, Chbib s'y installe et y tourne deux longs métrages avant de cofinancer Lana in Love (1991) auxquels on concède quelques moments comiques gâchés par un récit qui ne se maintient pas à la hauteur. Indubitablement Chbib est un passionné de cinéma qui tourne avec peu de moyens, mais dont les œuvres n'obtiennent pas aux États-Unis, comme au Canada, la reconnaissance qu'il souhaiterait.

Enfin les réalisateurs qui se font le plus remarquer proviennent de l'ONF qui s'avère, dans les années quatre-vingt, le principal foyer d'un cinéma personnel de qualité. Trois noms s'en dégagent. D'abord celui de Cynthia Scott dont The Company of Strangers (1990) séduit la presse par son ton, par ces petits moments inestimables qui en font le charme et par ses 
personnages riches et attachants. Mais ce sont surtout deux cinéastes qui opèrent ensemble à l'occasion, John N. Smith et Giles Walker, qui convainquent la critique. The Masculine Mystique (Smith \& Walker, 1984) est perçu avec plaisir comme un docu-drame qui applique à l'univers masculin les principes du féminisme. Les deux films suivants de Smith, Sitting in Limbo (1986) et Train of Dreams (1987) donnent l'occasion aux journalistes de dire beaucoup de bien du programme de fiction à l'ONF, qui produit des films si différents de ce que révèlent tant d'autres films canadiens. Train of Dreams surtout séduit, car il est réalisé et interprété avec tant de maîtrise et de réalisme qu'on a l'impression d'avoir affaire à un vrai documentaire. Pas étonnant que ce film achevé ait obtenu les Palmes d'Or au Festival international du film de Chicago et qu'on lui prédise un avenir honorable dans les salles d'art et d'essai.

Trois films de Walker bénéficient aussi de comptes rendus généralement favorables. 90 Days (1985) et The Last Straw (1987), qui forment une trilogie avec The Masculine Mystique, réussissent à faire rire à partir de situations simples et avec peu de moyens ${ }^{56}$. Pour une rare fois, ce fait renvoie à une appréciation positive. Après avoir ri grâce à un film " [...] unapologetically Canadian, with Alex touting his product (sperm) as Canadianmade and newscasters announcing that artificial insemination is sure to be a hot topic in the Canadian-U.S. free trade talks ${ }^{57}$ ", la presse trouve que Princes in Exile (1990) manque un peu cette fois d'inspiration bien qu'il demeure agréable à voir. À cause de son sujet — les jeunes qui souffrent du cancer —, on le destine au marché de la vidéo. N'eussent été ces six fictions produites par l'ONF, dont deux ont obtenu au Québec le prix L. E. OuimetMolson du meilleur long métrage de l'année, il faut reconnaître que le cinéma d'auteur anglo-québécois des 10 dernières années aurait fait piètre figure auprès de la critique des États-Unis.

Le nombre de films anglo-québécois recensés dans les répertoires du CRC/R étonne et plusieurs d'entre eux trouvent un écho dans la presse états-unienne. L'échantillonnage de publications retenu par le Centre biaise peut-être notre perception, mais il appert que ce sont les périodiques corporatifs (Variety, Box Office, The Hollywood Reporter) qui en recensent la plus 
grande partie. L'accueil critique du cinéma anglo-québécois aux États-Unis - et cela constitue une limite intrinsèque de notre étude - repose donc davantage sur les trade reviewers que sur les critiques de la presse régulière ou cinématographique.

On sait qu'il s'agit là de la partie la plus méprisée de l'institution journalistique au plan du discours, car sa fonction dérive davantage de la place de la publicité dans l'institution cinématographique que de celle de la critique. Autrement dit, alors que le journaliste de la presse quotidienne joue plutôt le rôle d'un guide du consommateur, celui de la presse corporative sert surtout à supputer et à évaluer les habitudes de fréquentation du public. Cette orientation "public" explique que les deux types de journalisme pratiquent principalement le tandem description/évaluation, négligeant pratiquement l'analyse. Le journalisme corporatif aligne davantage son discours évaluatif sur celui de l'industrie, il s'adresse donc au marché de la diffusion et de la promotion et pense dès lors en termes de débouchés. Ce fait explique notamment qu'abondent dans notre corpus les remarques quant à l'éventuel succès de tels films aux États-Unis ou quant à leur marché potentiel.

Dans son ensemble, la critique états-unienne ne formule pas de réserves contre le cinéma de genre, car celui-ci occupe une place capitale dans son propre cinéma ${ }^{58}$. Contrairement à la critique québécoise qui observe son cinéma national à travers le prisme de l'auteur et de la culture et qui a eu tendance à créer une antinomie entre cinéma d'auteur et cinéma de genre ainsi qu'à dévaloriser tout cinéma de genre produit au Québec, surtout $s$ 'il est en anglais, la critique états-unienne n'aborde pas la production anglo-québécoise avec de telles restrictions. A partir de l'éclosion d'un cinéma commercial canadien dans les années soixante-dix, elle procède de manière comparative. Elle juge ainsi le film anglo-québécois et canadien selon la valeur et les critères du cinéma états-unien. Le type de films avec lequel le cinéma québécois est surtout comparé, c'est la production lowbudget, de série $\mathrm{B}$ ou $\mathrm{Z}$, et la presse se montre sévère à son égard, le trouvant banal, mauvais, voire exécrable ${ }^{59}$.

En fait, quand le film n'est que banal sans être totalement mauvais, la presse nuance sa sanction et suggère généralement 
une distribution autre que dans les salles des grandes villes: dans les ciné-parcs, en région uniquement, à la télévision, à la télévision à péage ou, au pire, en home video. Par contre, si le film est bon, mais qu'il ne possède pas à ses yeux de potentiel chez le grand public, elle parlera plutôt du marché d'art et d'essai, des salles spécialisées ou de circuits des maisons d'enseignement. La presse indique clairement de cette manière la complexité du schéma de la distribution aux États-Unis, où plusieurs réseaux s'entrecroisent et se superposent, en même temps qu'elle démontre l'insignifiance de l'affirmation: "Le film a été vendu aux États-Unis." Il arrive d'ailleurs que les producteurs états-uniens trouvent largement leur compte en cofinançant ou en participant à des productions canadiennes. Comme l'explique un observateur :

An analysis of these models indicated clearly that international co-productions made with American participation enjoy the greatest success with a story set in Europe or Canada and involving genuine European or Canadian concerns. Canada surfaces as an important link for Americans in the co-production game. Canada has co-production treaties with many countries. Coproducing with a Canadian partner can provide U. S. producers an opportunity to participate at a level denied to them directly ${ }^{60}$.

La presse états-unienne réserve un traitement différent aux films étrangers qui traitent d'un sujet local et qu'on destine d'abord au marché local, et à ceux qui affichent d'office leurs prétentions internationales. Elle est souvent déçue par les films qui cachent leur côté canadien pour dépeindre un ailleurs sans personnalité où foisonnent les clichés. Quand ce cinéma essaie d'imiter Hollywood, elle se montre frustrée car, estime-t-elle, personne ne peut mieux réaliser un film hollywoodien que Hollywood. C'est pourquoi, engager des acteurs canadiens qui vivent à Hollywood ou des réalisateurs d'origine canadienne comme Arthur Hill, Ted Kotcheff et Daniel Petrie ne constitue à ses yeux qu'un piètre compromis ${ }^{61}$.

Cela dit, lorsqu'il y a trop de mauvais films en provenance du Canada, il arrive que des critiques, surtout ceux qui recherchent 
dans le cinéma réalisé au nord de leur frontière une certaine originalité et le signe d'une quelconque vitalité culturelle, se rebellent. Le commentaire suivant nous fournit un exemple d'une belle franchise:

Pinball Summer (George Mihalka, 1979) could be taken as a typical Canadian low-budget movie (which means, primarily, that it's shot without any Hollywood glamour stars). Jack Murphy, its bearded young producer, readily admits that he filmed it - for about $\$ 750,000$ - along the lines of an American-International youth-oriented action film [...]

There, I think, lies the problem for the young Canadian filmmaker today. Either they work the purely Quebec scene, for which there is at best a limited audience and scant financing, or they try to imitate the Hollywood movie and end up frustrated. Either way, they are left with nothing to build upon, nothing with which to set down the roots that might eventually flower into the Canadian equivalent of the young German cinema of today [...]

Adding to all this, I must also report, is a disturbing unwillingness on the part of the Canadians to support and encourage their own talents [...] The Canadian critics are notorious for their eagerness to wipe out anyone who dares to lift his head above the crowd ${ }^{612}$.

En fait, ce texte nous ramène au cœur de la production anglo-québécoise et, dans une moindre mesure, de la production canadienne. Depuis qu'ils s'occupent de réglementer les industries culturelles et de ramener le tout à des histoires de points de certification ${ }^{63}$ et à des clauses de traités, les gouvernements ont joué hypocritement sur l'ambiguïté et les glissements sémantiques, en portant préjudice au cinéma canadien de qualité. D'ailleurs, si les films dont nous avons traité brillent rarement par leur qualité artistique et culturelle, il semble arriver souvent qu'ils ne soient pas de si mauvaises affaires pour les investisseurs et les producteurs. C'est ici qu'une véritable histoire économique du cinéma au Québec nous éclairerait, ce à quoi les Canadiens anglais ont plus souvent porté attention ${ }^{64}$. Une bonne partie de la production canadienne n'existe que 
grâce à la politique fiscale et aux accords de coproduction conclus par le Canada, qui bénéficient principalement aux riches contribuables et aux firmes comptables et fiscales. N'y entrent presque exclusivement en jeu que des considérations de nature industrielle et commerciale: quand la SDICC prétend développer l'industrie cinématographique canadienne, elle devrait plutôt dire "au Canada", tout comme le célèbre contenu canadien de la télévision n'a souvent de canadien que l'emplacement du siège social ou la nationalité des interprètes et des principaux artisans. Le résultat patent de cette politique fut d'augmenter la masse monétaire investie au pays au détriment de la culture canadienne, aussi large que soit la définition de celle-ci. La réception critique états-unienne témoigne autant de la nature de ces produits que de la frustration qu'ils engendrent chez le spectateur le moindrement exigeant.

Si l'on compte sur le cinéma québécois de langue anglaise pour faire connaître et comprendre le Canada aux États-Uniens et aux étrangers, il est clair qu'on rate probablement la cible, car la majorité des critiques n'identifie pas la provenance des films, sauf pour dire tout le mal qu'il faut penser de ces piètres productions qui proviennent de ce minable Canada. Si l'on compte sur un débouché au sud de notre frontière pour permettre au cinéma de qualité de se rentabiliser et de faire sa marque, l'exception sera plutôt la règle, car les réseaux de distribution en salles sont plutôt hermétiques. Si l'on compte sur le même pays étranger pour rentabiliser des œuvres qui ont peu d'ambitions sauf celle de profiter des aides que consentent les États et de jouer la carte de l'effacement des distinctions pour fondre dans le grand melting pot hollywoodien, alors seulement peut-on parler d'un succès relatif.

D'ailleurs, peu importe la voie empruntée, nous serons toujours confinés aux marges de l'empire. La vie n'y est pas nécessairement désagréable et le rapport coûts/bénéfices, pas toujours si négatif. Du point de vue de la réception critique qui se dégage de notre corpus, force est de constater que, quand on est né pour un petit budget et qu'on est étranger, la langue ne constitue pas un facteur d'importance, car ce sont les critères valorisés par l'institution d'interprétation qui s'appliquent et ces critères 
tiennent rarement compte de celle-ci ou des états d'âme engendrés au Québec par le cinéma anglo-québécois. La réception de ces films permet donc de mettre en perspective toute la question de la réception du cinéma québécois aux États-Unis.

\section{Cinémathèque québécoise}

\section{NOTES}

1 Les numéros 24-25 de la revue Nouveau Cinéma canadien condensent pour la première fois, sous le titre "Bilan 1973 ", la production annuelle canadienne de long métrage. À partir du numéro 4 de la revue Copie Zéro intitulé "Annuaire longs métrages Québec 1978 ", la recension devient uniquement québécoise. En 1988, la revue cesse d'exister tandis que paraît dorénavant, sous forme de livre, l'Annuaire du cinéma québécois.

2 Possède le statut de québécois tout film financé en partie ou en totalité par des intérêts québécois, l'emplacement du siège social de la compagnie de production servant d'attestation.

3 La presse corporative et professionnelle comme Variety ou The Hollywood Reporter, quelques périodiques nationaux comme le New York Times ou le Time, et des revues de cinéma comme Cineaste ou CineFantastique.

4 Les répertoires du CRC/R comportent toujours deux parties selon cette ligne de division.

5 Ce sont parfois des correspondants non états-uniens qui écrivent ces textes. Par exemple, le critique québécois Jean-Pierre Tadros a longtemps été le correspondant canadien de Variety. Évidemment, j'ai exclu de mon corpus les textes qu'il avait publiés. Il y a aussi des Canadiens dont les textes paraissent dans des revues étatsuniennes. Eux aussi ont été exclus quand nous connaissions la nationalité de l'auteur.

6 À tel point qu'il n'est pas exagéré d'affirmer que l'étude de la réception des films anglo-québécois dans la presse états-unienne n'est qu'un sous-ensemble de la réception globale des films canadiens et que c'est à celle-ci qu'il faudrait s'attaquer.

7 Vingt ans plus tard, la suite de ce film, Unfinished Business (1984), sera reçue positivement à cause de l'énergie brute qui s'en dégage et de son ton d'une touchante sincérité.

8 Judith Crist, "Isabel", New York, 29 juillet 1968.

9 Article rédigé par un auteur dont le nom reste inconnu et publié dans la revue spécialisée Variety le 20 mai 1970.

10 Aux États-Unis comme au Canada, la notoriété de Paul Almond ira en déclinant. Ainsi sa grosse production de 6,4 millions \$, Final Assignment (1980), pourtant produite par une personne rompue aux arcanes de la production commerciale étatsunienne, Sandy Howard, fera à peine parler d'elle et n'a dû être distribuée que dans les circuits secondaires. Cette histoire, racontant la couverture, par une journaliste, de la visite à Moscou d'un premier ministre canadien, ne convainc personne: Montréal représente mal Moscou, le scénario est banal, les acteurs mauvais et la qualité technique inadéquate. Almond essaiera d'explorer une autre direction en réalisant à Vancouver une comédie pour adolescents, Ups \& Downs (1983). L'accueil sera poli, à la mesure d'un réalisateur devenu impersonnel. Ses deux films ultérieurs ne sont pas recensés dans les journaux dépouillés par le CRC/R. 
11 Charles Lazarus, "Montreal Begins To Hope Films Have Future With Canadian Aid; Divides Over, Excited By, Almond. ", Variety, 30 septembre 1970, p. 13.

12 Quelques films des années soixante-dix vont essayer de jouer la carte de la qualité. Pensons à Why Rock the Boat (John Howe, 1974), produit par l'ONF et qui reçoit un Hugo de bronze au Festival de Chicago. Certains trouvent que cette gentille comédie manque de personnalité. En réalité, la presse états-unienne ne recherche pas nécessairement une qualité propre ou conventionnelle, pas plus qu'elle ne s'enthousiasme pour des sujets dont l'impact chez elle n'apparaît pas toujours clairement, comme c'est le cas pour Two Solitudes (Lionel Chetwynd, 1977). En fait, les chances de succès de la qualité "à la canadienne" aux États-Unis seront plutôt minces.

13 "A. B. P. Looks To Hollywood To Set Co-Production Deals", The Independent Film Journal, 24 décembre 1973, p. 16. Cette déclaration reprend presque mot à mot ce qui va paraître dans le numéro 12 de Cinema Canada en 1974. Voir aussi à ce sujet "English Language Films in Montreal", Canadian Film Digest (février 1973).

14 "U. S. Distrib Deals For Canadian Films Are On An Upswing", Variety, 28 mai 1980 , p. 7.

15 "The Pyx", The Independent Film Journal, 12 novembre 1973, p. 20-21.

16 L'extrême difficulté de la critique et des historiens québécois à considérer comme cinéastes québécois ces Canadiens qui n’habitent pas le Québec mais viennent y réaliser leurs films fait en sorte qu'il faudra attendre la deuxième édition du Dictionnaire du cinéma québécois pour que le nom de Cronenberg y figure.

17 Ron Pennington, "Death Ship", The Hollywood Reporter, 17 avril 1980, p. 7.

18 David Linck, "Happy Birthday to Me", Box Office, n 117 (1981), p. 51.

19 Jean Lafleur avait conçu l'histoire initiale et devait réaliser le film. Son ineptie et son amateurisme sont tellement flagrants qu'on le met à la porte après quelques jours. Un tel budget oblige les producteurs à plus d'exigences que pour les navets habituels.

20 Certains journalistes canadiens ont déjà noté que le sort réservé par Hollywood à la représentation des Canadiens - dont témoigne abondamment le livre de Pierre Berton Hollywood's Canada. The Americanization of Our National Image (Toronto: McClelland/Stewart, 1975) - était peu de chose à côté du sort réservé par les Canadiens eux-mêmes à leurs propres réalisations.

21 Il faut mentionner que Lord s'est dissocié du film en précisant qu'il s'agissait d'une œuvre de commande.

22 Lor. "Eddie and the Cruisers II", Variety, 16 août 1989.

23 Le sujet d'ailleurs est $100 \%$ états-unien: l'action se passe en Floride dans les années cinquante et tourne autour des angoisses sexuelles de la jeunesse de l'époque.

24 Le film obtient plus de 40 millions $\$$ de recettes à sa sortie sur plus de 1500 écrans alors qu'il en a coûté 10 fois moins. On parle de plus de 100 millions \$ l'année suivante, ce qui est énorme pour un film coté « R». Cela représente un rapport coût/ recette de 40.

25 Le public qui fréquente les salles commerciales ne sera pas dupe, la sortie du film dans 129 salles en novembre et décembre 1983 ne rapportant qu'un pauvre $214000 \$$.

26 Comme ils ne le peuvent pas en comédie musicale, ainsi que le démontre l'exemple de Hey Babe! (Rafal Zielinsky, 1983).

27 Ilsa, She Wolf of the S. S. (Don Edmonds, 1973), Ilsa, Harem Keeper of the Oil Sheiks (Don Edmonds, 1975), Ilsa, the Tigress of Siberia (Ivan Reitman, 1977).

28 Ce film est un des quatre longs métrages canadiens produits en 1983 grâce au soutien financier de Playboy Channel qui, après les avoir présentés à la télévision, les rendra disponibles en vidéocassettes en 1986. 
29 Heureusement qu'un film comme Not a Love Story: A Film About Pornography (Bonnie Sherr Klein, 1981) donne un autre son de cloche sur la représentation de la femme et son exploitation. Mais la majorité de la presse, sans contester l'honnêteté de la cinéaste, ne souscrit pas à son point de vue féministe, car il n'est pas simple de départager l'érotisme de la pornographie. Le film a droit à une lecture politique assez surprenante puisque la critique soutient que l'identité canadienne du film explique la dénonciation de la pornographie comme étant une autre manifestation de l'impérialisme culturel états-unien.

30 Il convient de rappeler que c'est en 1981 que les neuf plus importantes compagnies de production du Canada se regroupent au sein de l'Association canadienne des maisons de production cinématographique où dominent les firmes québécoises International Cinema Corporation, RSL Films, Ronald I. Cohen Productions, Filmplan International, DAL Productions, Astral Bellevue Pathé. Les films qu'elles concoctent visent essentiellement le marché états-unien et plusieurs de leurs productions bénéficient de l'apport des Majors (Columbia, 20th Century-Fox) et des Mini-Majors (Avco-Embassy).

31 Compagnie fondée en 1969 par John Kemeny avec Joseph Koenig et Donald Duprey, deux anciens de l'ONF. Dix ans plus tard, Kemeny s'associe à Denis Héroux pour créer un autre ICC, International Cinema Corporation. C'était la Columbia qui avait distribué Shawdow of the Hawk (George McGowan, 1976) et White Line Fever.

32 Cette habitude agace plus d'un États-Unien. Ainsi, à propos de Harry Tracy, Desperado (William A. Graham, 1981) produit par la compagnie montréalaise Ronald I. Cohen Production, un critique écrira: "A Canadian production, wanting it in all respects to look like an American movie. Filming was done on British Columbia locations. But British Columbia is posing as Wyoming and the film's hero and theme is ultra-American. The mixture is meant to help film slip into U.S. theatres without its foreign origin being noticed. But as in many earlier instances of Canadian filmmaking, the brew just will not come to a boil " (Kell. "Harry Tracy, Desperado", Variety, 27 janvier 1982).

33 A preuve, ce commentaire sur Bayo (Mort Ransen, 1984) : "While other Canadian filmmakers were masquerading the streets of Toronto for those of Toledo and attempting third-rate spinoffs of Beach Blanket Bingo and the like during the infamous Canadian tax shelter days of the late '70s, Montreal producer Harry (Lies My Father Told $\mathrm{Me}$ ) Gulkin stuck to Canadian culture themes and carried the hopes of many that Canada could in fact develop its own distinct and vibrant film identity... Bayo is as Canadian as the country's famed "goose " and its maple syrup" (Bro. "Bayo", Variety, 8 mai 1985).

345300000 \$, en cofinancement avec Sandy Howard Productions de Los Angeles.

35 Par contre, sa présence dans The Blood of the Others (1984) et The Hotel New Hampshire (1984) s'explique alors par son statut de vedette.

36 Le vedettariat étranger et le style de film qu'il impose coûte cher, très cher. Par exemple, Agency commandera un budget de 4,4 millions \$. Pour atteindre cet objectif, Robert Lantos et Stephen Roth seront les premiers à faire appel à l'investissement public et à publier un prospectus à cet effet.

37 Sa première apparition dans un film canadien date de 1969 dans The Act of the Heart. On le voit plus fréquemment à partir de 1977 quand le cinéma commercial canadien jouera de plus en plus la carte du vedettariat. En ce qui concerne le cinéma anglo-québécois, il sera notamment le partenaire de Susan Anspach dans Gas (Les Rose, 1980), il incarnera un membre du politburo dans Eminent Domain (John Irvin, 1990) avant de participer à plusieurs coproductions, Bethune: The Making of a Hero (Philip Borsos, 1990), Scream of Stone (Werner Herzog, 1991) et Buster's Bedroom (Rebecca Horn, 1991).

38 Ce Torontois tournera dans les années soixante-dix dans six longs métrages ontariens avant de connaître le succès aux États-Unis. 
39 Cette Montréalaise va se faire connaître surtout en Europe au début des années soixante. Son premier film québécois sera The Uncanny (Denis Héroux, 1977) et on la verra régulièrement pendant une dizaine d'années.

40 Natif de Québec, il travaille très tôt aux États-Unis. On le retrouve dans quelques films québécois anglophones - Joshua Then and Now (Ted Kotcheff, 1985), Keeping Track (Robin Spry, 1986), Malarek (Roger Cardinal, 1988), The Phone Call (Allan A. Goldstein, 1989) - et même dans une production francophone, La Florida (Georges Mihalka, 1993).

41 Voir à ce sujet le chapitre III du livre de Louise Carrière, Les Relations cinématographiques France-Québec (Montréal : Centre de recherche cinéma/réception et Cinémathèque québécoise, 1994). Des 17 coproductions franco-canadiennes tournées en anglais entre 1980 et 1990, huit sont réalisées par des Français.

42 Une coproduction France-Canada-Israël censée se dérouler en Afrique et mettant en vedette Tony Curtis.

43 Ce film a été tourné en deux versions, française et anglaise.

44 Autre cas curieux accueilli avec indifférence: le film en cofinancement canadoaméricain Hot Touch (1981), tourné par Roger Vadim et mettant en vedette MarieFrance Pisier.

45 Célèbre photographe et artiste depuis les années cinquante, Frank donne ici un road movie que la presse accueille avec plaisir en évoquant Jim Jarmush et d'autres cinéastes indépendants new-yorkais.

46 Jim Robbins, "Atlantic City", The Film Journal, vol. 84, n" 6 (1981), p. 13.

47 Andrew Sarris, "Wit and Charm on the Boardwalk", The Village Voice, $1^{\text {"t }}$ avril 1981 , p. 49.

48 Cronenberg a connu le même sort, les critiques ayant déjà nommé Scanners le meilleur film américain de 1981 .

49 Amorcé aux États-Unis en 1978-1979, le film bloque à cause de la grève de juillet 1980. On le propose alors aux producteurs canadiens. Annaud n'a pas tellement apprécié l'expérience, s'étant plaint d'avoir dû consentir à des sacrifices créatifs et personnels pour se plier aux règles canadiennes en ce qui a trait au nombre d'acteurs et de techniciens canadiens à embaucher, qui n'étaient pas toujours compétents.

50 Voir Jones, Edward T., "Checking into While Others Run to Check Out of Tony Richardson's The Hotel New Hampshire ", Literature/Film Quarterly, vol. 13, n"1 (1985), p. 66-69.

51 Il y a une certaine ironie à ce qu'un film parlé en anglais obtienne ce prix. Aux yeux des Américains, l'anglais hors Êtats-Unis est peut-être une langue étrangère.

52 Il faut réserver une place spéciale à Ticket To Heaven (Ralph L. Thomas, 1981), tourné à Toronto pour une compagnie de Montréal. Cette remarquable dénonciation du fanatisme religieux inspirée de Moon obtient de longs textes enthousiastes dans Cineaste et le New York Times en plus d'un accueil généralement positif comme l'indique la citation suivante: "This hard-hitting docu-drama ranks as one of the most outstanding films to emerge from Canada in recent years and should have no problem finding international distribution and audiences" (Klad., "Ticket To Heaven", Variety, 27 mai 1981).

53 Il faut rappeler que né à Budapest en 1933, Kaczender est entré à l'ONF en 1957. Par ailleurs $U$-Turn (1973) lui avait déjà valu un accueil négatif lors de sa présentation au Festival d'Atlanta en 1973.

54 Il y a aussi le cas de Danièle J. Suissa, qui réalise comme premier long métrage The Morning Man (1986). À cause d'un scénario et de dialogues insipides ainsi que d'une musique trop emphatique, le film ne convainc pas la critique, qui ne lui prédit 
un salut qu'en vidéocassette. D'ailleurs, Suissa tournera la majorité de ses films ultérieurs pour la vidéo ou la télévision.

55 Voir Strat., "Evixion", Variety, 3 septembre 1986. Précisons que cet auteur se trompe, car la seule aide de l'État obtenue par le film est celle du programme PAFFPS de l'ONF, ce qui ne constitue pas un véritable investissement.

56 Le premier obtient d'ailleurs au Festival de Chicago une "Gold Plaque, Feature Film Category for innovation method of creating film comedy ".

57 Devo, "The Last Straw", Variety, 23 septembre 1987.

58 Il faut noter aussi que la critique interprétative et analytique, tout comme la réflexion savante, ont procuré à l'étude du cinéma de genre d'importantes lettres de noblesse.

59 Le portrait aurait pu être plus accablant si je m'étais arrêté aux opinions émises pour des films comme Yesterday (Larry Kent, 1979), Crunch (Mark Warren, 1980), Hog Wild (Les Rose, 1980), Gas (Les Rose, 1980), The High Country (Harvey Hart, 1980), Tulips (Rex Bromfield, 1981), Breaking All the Rules (James Orr, 1985), Crazy Moon (Allan Eastman, 1986), The Carpenter (David Wellington, 1987) et autres Blind Fear (Tom Berry, 1989).

60 Thomas Selz, "Ecco! Ecco!", The Off-Hollywood Report, avril-mai 1990, p. 18.

61 Les abris fiscaux encouragent ces pratiques et on y retrouve un nombre exceptionnel de films anglo-canadiens mettant en vedette des acteurs américains et réservant aux Canadiens les rôles de soutien, à moins que ces derniers n'aient déjà une carrière internationale.

62 Arthur Knight, "Canadian Films: A Suicide Course", The Hollywood Reporter, 14 septembre 1979 , p. 10 et 28.

63 Une des escroqueries notoires pour augmenter indûment le nombre de points fut d'avoir eu largement recours aux producteurs délégués qui n'étaient finalement que les rois-nègres des producteurs américains.

64 Il suffit de se rappeler la place que la regrettée revue Cinema Canada accordait au volet "production" du cinéma canadien. Il existe aussi quelques ouvrages anglophones qui approfondissent la question. Outre l'ouvrage de Ted Magder, Canada's Hollywood. The Canadian State and Feature Films (Toronto: University of Toronto Press, 1993), signalons celui de Manjunath Pendakur, Canadian Dreams of American Control. The Political Economy of the Canadian Film Industry (Toronto: Garamond Press, 1990). Ils expliquent en détail l'impact des mesures économiques et fiscales sur la production et permettent par exemple de comprendre pourquoi, vers 1974, Astral conclut une entente avec Columbia pour être sous-distributeur pour les salles indépendantes tandis que son bureau de Toronto fait affaire avec Odeon; cet accord explique peut-être les mesures de réciprocité dans la production et distribution de films Astral aux États-Unis.

\section{OUVRAGES CITÉS}

Bérubé, Bernard et Richard Magnan. Les Films québécois dans la critique américaine. Répertoire analytique 1960-1979. Montréal : Centre de recherche cinéma/réception et Cinémathèque québécoise, 1994.

Bérubé, Bernard et Richard Magnan. Les Films québécois dans la critique américaine. Répertoire analytique 1980-1992. Montréal : Centre de recherche cinéma/réception et Cinémathèque québécoise, 1994.

Bérubé, Bernard et Richard Magnan. Les Films québécois aux États-Unis (1980-1991). Montréal : Centre de recherche cinéma/réception et Cinémathèque québécoise, 1994. 
Bordwell, David. Making Meaning. Inference and Rhetoric in the Interpretation of Cinema. Cambridge: Harvard University Press, 1989.

Carrière, Louise. Les Relations cinématographiques France-Québec. Montréal: Centre de recherche cinéma/réception et Cinémathèque québécoise, 1994.

Magder, Ted. Canada's Hollywood. The Canadian State and Feature Films. Toronto: University of Toronto Press, 1993.

Pendakur, Manjunath. Canadian Dreams of American Control. The Political Economy of the Canadian Film Industry. Toronto: Garamond Press, 1990.

Turner, D. John. Canadian Feature Film Index/Index des films canadiens de long métrage 1913-1985. Ottawa: Archives publiques du Canada, 1987. 